\title{
Methodology for modeling of city sustainable development based on fuzzy logic: a practical case
}

\begin{abstract}
Information on sustainability can be used for future development planning. This study presents an approach for assessing urban sustainability. Delphi and fuzzy logic methods and the Kruskalï Wallis test were the discovery and verification techniques used. The city system comprised social, economic, and environmental subsystems. The seven orientors of existence, effectiveness, freedom of action, security, adaptability, coexistence, and psychological need were measured using different indicators. The final sustainability output was obtained by aggregation of the multiple orientors and subsystems sustainability values into a unified measure. A fuzzy sustainability index was developed to compare the importance of the sustainability orientors and subsystems. The model was applied to Mahshahr, an industrialized coastal city in Iran. The model output for the subsystems showed significant differences between the economic and environmental subsystems and the social subsystem. The final sustainability output showed that the effectiveness orientor gave the highest sustainability value. The model is dynamic and can be modified for different purposes by changing the indicators. With this model, policy-makers can evaluate existing city sustainability and predict future sustainability by varying the indicators. This can be done on local, regional, and global scales for security and adaptation strategies, mitigation plans, and sustainable development management.
\end{abstract}

Keyword: Fuzzy sustainability index; Orientor; Sustainability; Sustainable development 